\title{
RETROGRADE LEFT VENTRICULAR CARDIOANGIOGRAPHY IN THE DIAGNOSIS OF MITRAL REGURGITATION
}

\author{
BY
}

\author{
I. R. GRAY, C. S. JOSHIPURA, AND J. MACKINNON \\ From Birmingham Regional Thoracic Centre, Hertford Hill, Nr. Warwick
}

Received July 24, 1962

Accurate diagnosis is often difficult when signs of mitral stenosis and regurgitation coexist or when the presence of a tricuspid or aortic systolic murmur suggests mitral regurgitation in a patient with pure stenosis. Many techniques of investigation have been applied to this problem but have failed to improve greatly upon the accuracy of bedside diagnosis. Pre-operative diagnosis must be as exact as possible to avoid unnecessary exploration of the mitral valve and to select patients for the surgical correction of mitral regurgitation. Direct measurement of the left atrial pressure has given helpful information, but the interpretation of the pressure curves is difficult, particularly when there is atrial fibrillation. Cardioangiography after injection of contrast medium into the left ventricle can demonstrate the presence and degree of mitral regurgitation as well as giving information about the size and function of the left atrium and ventricle.

Contrast medium can be injected into the left ventricle either through a needle (Ponsdomenech and Beato Núñez, 1951) or through a catheter passed from the aorta (Prioton et al., 1957) or from the left atrium (Brockenbrough, Braunwald, and Ross, 1962). Cardioangiography by means of left ventricular puncture has been employed successfully in the study of mitral regurgitation (Smith, Cregg, and Klassen, 1956; Lehman, Musser, and Lykens, 1957; Björk, Lodin, and Malers, 1960) but retrograde arterial catheterization has been preferred as being safer and easier by Dotter and Gensini (1960) and Amplatz et al. (1961). This method has the advantage of providing pressure tracings over the aortic valve.

We have carried out retrograde arterial left ventricular cardioangiography in an attempt to improve the accuracy of our diagnosis of mitral valve disease and to select patients for closed and open mitral valve operations. It has been done in conjunction with other methods of clinical assessment in order to judge their value and it has been possible to compare the results with surgical or post-mortem findings in several patients.

\section{SubJeCTS AND MethodS}

Thirty patients with mitral valve disease were studied. All were seriously disabled and operation was deemed necessary. Mitral regurgitation was thought to be present because of a systolic murmur in every case and evidence of left ventricular hypertrophy in the majority. Disease of the aortic valve was present in 14 patients and tricuspid valve disease in 4 . Conventional right cardiac catheterization was carried out, a wedged pulmonary arterial pressure being recorded if possible: the $\mathrm{Ry} / \mathrm{v}$ ratio (Owen and Wood, 1955) was calculated from this. The brachial artery or one of its branches was then exposed through the same incision in the ante-cubital fossa. A flexible thin-walled nylon catheter (No. 6 or 7 N.I.H. pattern) was introduced through a longitudinal incision in the artery. It passed into the ascending aorta without difficulty in every case. The passage of the catheter tip through the aortic valve was accomplished by advancing it during systole, varying its direction by rotation, and sometimes by forming a loop in the ascending aorta. 
Entry into the ventricle was recognized by the position of the catheter, and by the pressure pulse which was recorded continuously.

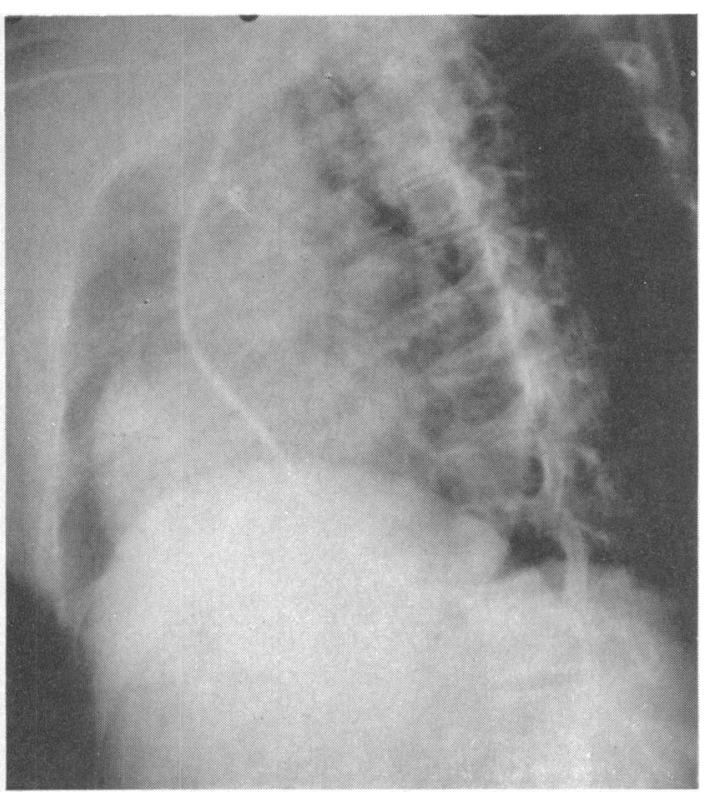

FIG. 1.-Preliminary film in right posterior oblique position showing opacified catheter in left ventricle. The catheter tip is too far down and is touching the endocardium at the apex, and the course of the catheter is rather tortuous. The catheter was withdrawn $7 \mathrm{~cm}$. before contrast medium was injected.
Exposures could only be made in a single plane with our apparatus and a right posterior oblique position was selected as giving a good view of the left atrium, left ventricle, and aorta, as well as the mitral valve itself (Smith et al., 1956). This position also made slight opacification of the left atrium easy to detect by comparison with the non-opacified right ventricle which lies anterior. The catheter was opacified by injecting a small amount of contrast while the preliminary film was exposed. Its position was then adjusted so that the tip was low in the ventricular cavity but clear of its wall (Fig: 1). If the course of the catheter in the aorta appeared tortuous it was withdrawn sufficiently to take up the undulations. The patient was rotated about $60^{\circ}$ from the antero-posterior position, the degree of rotation being adjusted in each case to clear the left cardiac chambers from the aorta and the spine.

When the final alterations of the patient's position and the catheter had been made the patient was anæsthetized: $35-40 \mathrm{ml} .85$ per cent hypaque were injected using a Talley pressure injector set at $100 \mathrm{lb}$./in. ${ }^{2}$, and the films were exposed immediately at a rate of four films per second for the first 4 to 6 seconds and less frequently for 6 seconds afterwards. The injection itself usually lasted between 1.5 and 3 seconds. Electrocardiogram was recorded throughout. A pressure recording was made as the catheter was withdrawn from the left ventricle to the aorta if aortic valve disease was suspected. The catheter was then removed and the artery repaired with atraumatic silk sutures.

The severity of mitral regurgitation was judged from the degree of left atrial opacification and from comparison of left atrial and aortic filling. It was assessed in four grades of severity according to the density of contrast in the left atrium and the size of the chamber (Fig. 2). Thus, slight opacification of a small left atrium was grade 1 (Fig. 3), dense opacification of a large atrium, grade 4 (Fig. 4), with grades 2 and 3 lying between.

Björk et al. (1960) pointed out that comparison of the filling of the aorta with the left atrium indicated the proportion of the left ventricular output that flowed back into the atrium (Fig. 5). Patients were placed in Group A with severe regurgitation, when the left atrium was completely filled before contrast reached the top of the aortic arch, in Group B with moderate regurgitation, when contrast was half-way down the thoracic aorta, and in Group $C$ with slight regurgitation, when the whole of the thoracic aorta was filled when the left atrium was completely opacified.

The radiographic appearance of the mitral valve was carefully scrutinized in systolic and diastolic films and the length and breadth of the ventricular cavity were measured in systole and diastole.

\section{RESULTS}

The procedure was completed successfully in 24 of the 30 patients. It was abandoned in 1 patient because of electrocardiographic changes due to catheterization of a coronary artery. The catheter could not be passed across the aortic valve in 2 patients, and in 1 it slipped back into the aorta during the final positioning of the patient and the contrast medium was injected into the aorta. Serious ventricular arrhythmias occurred in 2 patients when the catheter entered the left ventricle, and no contrast medium was injected. The degree of mitral regurgitation could be assessed with 
GRADE I

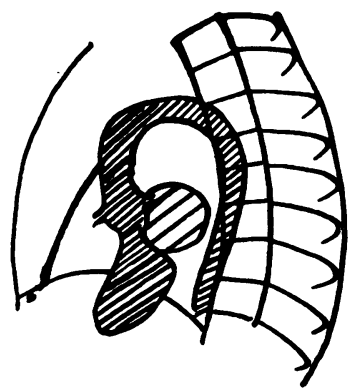

GRADE 4

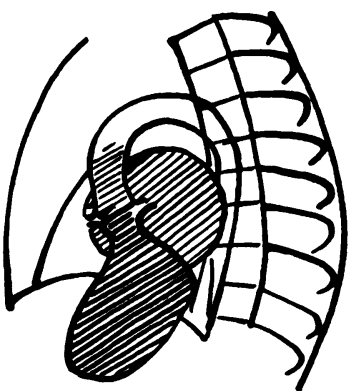

FIG. 2.-Diagrammatic illustration of the diagnostic features in the assessment of mitral regurgitation by cardioangiography. The important features are the filling of the left atrium and left ventricle and the degree of opacification of the left atrium and of the aorta. In grade 1 mitral regurgitation there is slight opacification of a small left atrium and early filling of the entire aorta: in grade 4 mitral regurgitation there is dense opacification of a large left atrium and filling of the aorta is delayed.

confidence from examination of the angiogram in all 24 cases. Two were judged to have no regurgitation. Opacification of the left atrium was demonstrated in the remaining 22, and was estimated as grade 1 in 5 patients, grade 2 in 7 , grade 3 in 9 , and grade 4 in 1 . These assessments are compared with the Björk classifications (see Table). When gross regurgitation was suspected on clinical grounds this was usually confirmed by the investigation but in the majority of patients the physical signs had given rise to doubt so that comparison of the radiological with clinical conclusions was not possible.

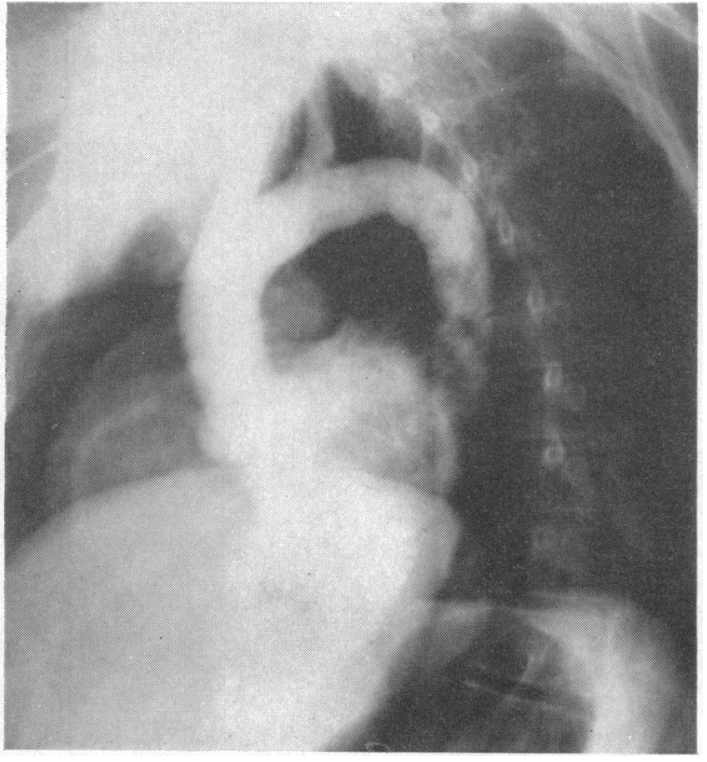

FIG. 3.-Grade 1 mitral regurgitation showing a jet of contrast medium filling the upper part of quite a small left atrium. Slight regurgitation with severe mitral stenosis was found at valvotomy.

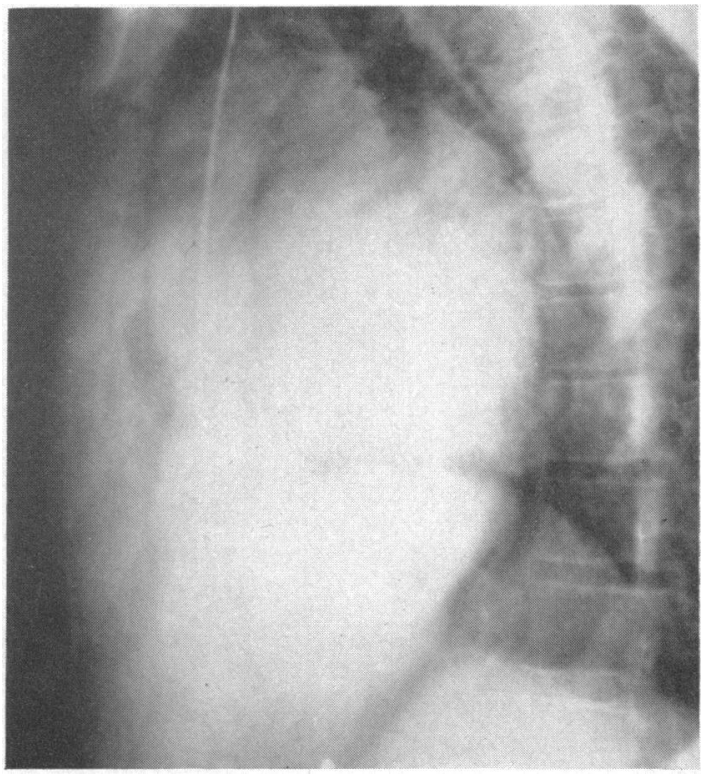

Fig. 4.-Grade 4 mitral regurgitation showing dense opacification of an enormous left atrium. The left ventricle is also very large and calcification of the mitral valve can be seen between the two chambers. 


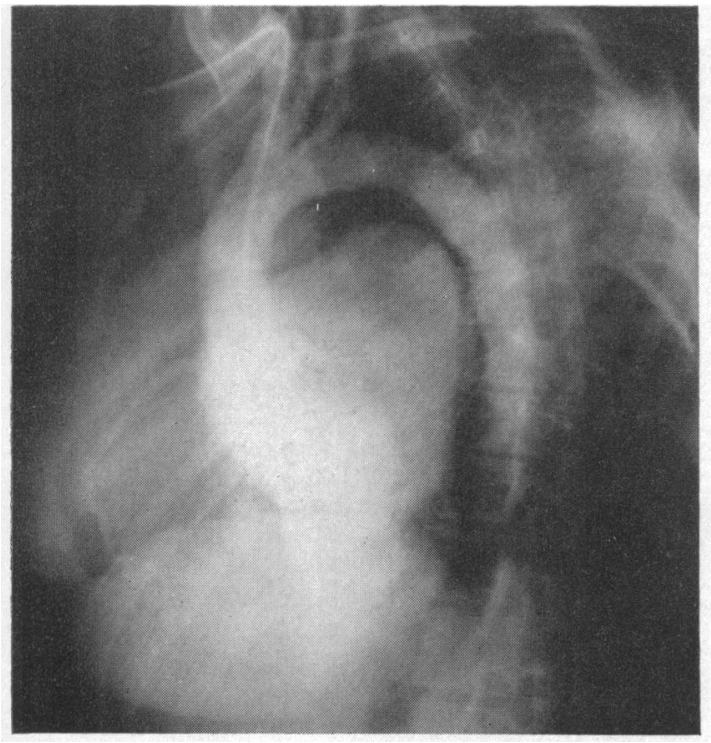

FIG. 5.-Grade 3 mitral regurgitation with dense opacification of a large left atrium. Contrast medium has filled the upper half of the thoracic aorta when left atrial filling is complete so that this patient is in Björk's Group B. Severe mitral regurgitation was confirmed at operation.

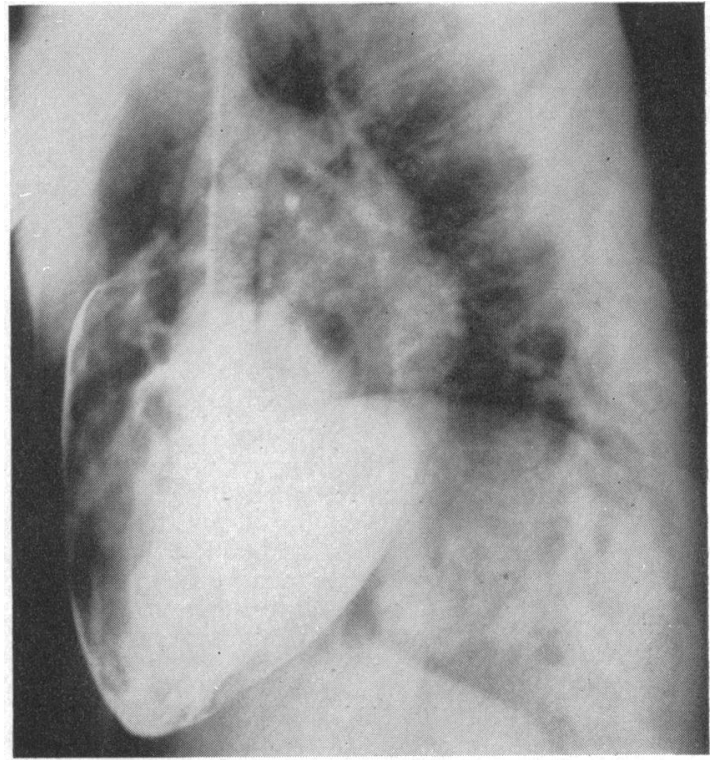

Fig. 6.-Left ventricular cardioangiogram showing contrast medium penetrating the myocardium and outlining the pericardial cavity. The catheter tip can be seen at the apex of the left ventricle.

The appearance of the mitral valve varied widely. Where there was severe mitral regurgitation the atrium and ventricle were virtually continuous and in many cases there seemed to be little change in the appearance of the mitral valve region throughout the cardiac cycle. Ballooning of the mitral valve into the ventricle during diastole, which was described by Arvidsson (1958) in angiograms after right-sided injection of contrast medium, was judged to be present in seven patients. Two of these had no regurgitation and the other five had mitral regurgitation of grades 1 to 3 . Its presence was not correlated closely with a loud first heart sound or opening snap although it does seem likely to indicate mobile and undistorted valve cusps.

There appeared to be a jet of contrast from the ventricle to the atrium in three patients with slight mitral regurgitation (Fig. 3). This appearance did not have any diagnostic significance. The size of the left ventricle could readily be judged from the cardioangiograms and its volume was estimated approximately in each case. This assessment helped in the overall appreciation of cases but was not consistently related to the degree of mitral regurgitation.

The results of cardioangiography were compared with various other forms of clinical assessment. Patients were divided into three groups: no mitral regurgitation, grades 1 and 2 regurgitation, and grades 3 and 4 regurgitation. The correlation between the degree of regurgitation and various clinical observations was disappointing and reflects the difficulties in diagnosis of this type of case. The findings in the small group with no regurgitation cannot be regarded as significant for these were atypical examples of mitral stenosis in patients with multivalvular disease. Slight differences in the auscultatory signs were found between those with lesser and greater degree of mitral regurgitation. A loud first heart sound was only found in 3 of 10 patients with grade 3 or 4 mitral regurgitation while it was recorded in 9 out of 12 with lesser degree of regurgitation. The mitral systolic murmurs tended to be louder the greater the regurgitation. A grade 4 (out of 6) murmur was found in 5 out of 10 patients with grade 3 or 4 regurgitation and in only 2 out of 12 with grade 1 and 2 regurgitation. 
TABLE

\begin{tabular}{|c|c|c|c|c|c|c|c|c|c|c|c|}
\hline \multirow{2}{*}{\multicolumn{2}{|c|}{$\begin{array}{r}\text { Cardic } \\
\text { as }\end{array}$}} & \multirow[b]{2}{*}{ Patient } & \multirow[b]{2}{*}{ 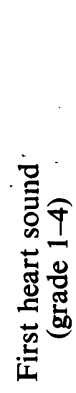 } & \multirow[b]{2}{*}{ 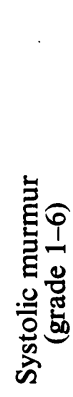 } & \multirow[b]{2}{*}{ 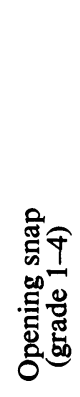 } & \multicolumn{2}{|c|}{$\begin{array}{l}\text { Diastolic } \\
\text { murmur }\end{array}$} & \multirow[b]{2}{*}{ 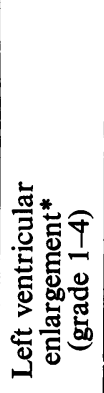 } & \multirow[b]{2}{*}{$\begin{array}{l}\stackrel{\circ}{\pi} \\
\frac{2}{2} \\
\frac{2}{\alpha}\end{array}$} & \multirow[b]{2}{*}{$\begin{array}{l}\text { 号 } \\
\text { 品 } \\
\text { : } \\
\text { : }\end{array}$} & \multirow[b]{2}{*}{ 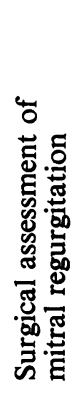 } \\
\hline & & & & & & 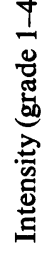 & 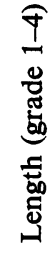 & & & & \\
\hline \multicolumn{2}{|c|}{$\begin{array}{l}\text { No mitral } \\
\text { regurgitation }\end{array}$} & $\begin{array}{l}\text { B.B. } \\
\text { M.W. }\end{array}$ & $\stackrel{2}{\mathrm{~N}} \dagger$ & $\begin{array}{l}3 \\
3\end{array}$ & $\begin{array}{l}2 \\
1\end{array}$ & $\begin{array}{l}1 \\
4\end{array}$ & $\begin{array}{l}1 \\
3\end{array}$ & $\begin{array}{l}1 \\
1\end{array}$ & $\overline{0.35}$ & 二 & $\begin{array}{l}\mathbf{0} \\
\mathbf{0}\end{array}$ \\
\hline \multirow{4}{*}{ 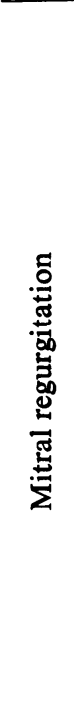 } & Grade 1 & $\begin{array}{l}\text { E.M. } \\
\text { A.C. } \\
\text { A.Q. } \\
\text { G.B. } \\
\text { F.W. }\end{array}$ & $\begin{array}{l}1 \\
2 \\
1 \\
1 \\
2\end{array}$ & $\begin{array}{l}3 \\
1 \\
3 \\
3 \\
4\end{array}$ & $\begin{array}{l}2 \\
0 \\
0 \\
2 \\
2\end{array}$ & $\begin{array}{l}3 \\
3 \\
2 \\
3 \\
3\end{array}$ & $\begin{array}{l}2 \\
3 \\
2 \\
3 \\
2\end{array}$ & $\begin{array}{l}1 \\
1 \\
1 \\
1 \\
1\end{array}$ & $\begin{array}{l}0.6 \\
1.7 \\
0.5 \\
0.7\end{array}$ & $\begin{array}{l}\mathbf{C} \\
\mathbf{C} \\
\mathbf{B} \\
\mathbf{C} \\
\mathbf{B}\end{array}$ & $\begin{array}{l}1 \\
1 \\
1 \\
-\end{array}$ \\
\hline & Grade 2 & $\begin{array}{l}\text { R.H. } \\
\text { A.E. } \\
\text { R.B. } \\
\text { J.W. } \\
\text { K.L. } \\
\text { M.W. } \\
\text { E.S. }\end{array}$ & $\begin{array}{l}\mathbf{N} \\
1 \\
3 \\
2 \\
\mathbf{N} \\
3 \\
1\end{array}$ & $\begin{array}{l}3 \\
4 \\
3 \\
3 \\
3 \\
1 \\
3\end{array}$ & $\begin{array}{l}0 \\
2 \\
3 \\
2 \\
0 \\
1 \\
0\end{array}$ & $\begin{array}{l}2 \\
3 \\
2 \\
3 \\
4 \\
2 \\
2\end{array}$ & $\begin{array}{l}3 \\
2 \\
2 \\
3 \\
3 \\
3 \\
2\end{array}$ & $\begin{array}{l}1 \\
2 \\
0 \\
2 \\
1 \\
3 \\
1\end{array}$ & $\begin{array}{l}1.9 \\
1.6 \\
1.1 \\
0.8 \\
1.8 \\
1.5\end{array}$ & $\begin{array}{l}\text { C } \\
\text { B } \\
\text { B } \\
\text { A } \\
\text { C } \\
\text { C } \\
\text { B }\end{array}$ & $\begin{array}{l}2 \\
2 \\
1 \\
3 \\
0 \\
\end{array}$ \\
\hline & Grade 3 & $\begin{array}{l}\text { J.C. } \\
\text { E.T. } \\
\text { S.W. } \\
\text { V.L. } \\
\text { M.E. } \\
\text { I.B. } \\
\text { J.D. } \\
\text { A.C. } \\
\text { A.B. }\end{array}$ & $\begin{array}{l}\mathbf{N} \\
3 \\
\mathbf{N} \\
\mathbf{N} \\
2 \\
2 \\
\mathbf{N} \\
\mathbf{N} \\
\mathbf{N}\end{array}$ & $\begin{array}{l}3 \\
3 \\
4 \\
4 \\
4 \\
2 \\
4 \\
4 \\
3\end{array}$ & $\begin{array}{l}0 \\
0 \\
0 \\
0 \\
2 \\
2 \\
0 \\
0 \\
1\end{array}$ & $\begin{array}{l}4 \\
0 \\
3 \\
3 \\
4 \\
1 \\
1 \\
2 \\
3\end{array}$ & $\begin{array}{l}3 \\
0 \\
2 \\
2 \\
3 \\
1 \\
2 \\
1 \\
3\end{array}$ & $\begin{array}{l}1 \\
1 \\
2 \\
2 \\
1 \\
2 \\
3 \\
2 \\
1\end{array}$ & $\begin{array}{l}0.7 \\
1.8 \\
2.2 \\
0.8 \\
3.2 \\
1.6 \\
=\end{array}$ & $\begin{array}{l}\mathbf{A} \\
\mathbf{C} \\
\mathbf{B} \\
\mathbf{A} \\
\mathbf{B} \\
\mathbf{C} \\
\mathbf{A} \\
\mathbf{A} \\
\mathbf{B}\end{array}$ & $\begin{array}{l}\frac{-}{3} \\
\frac{-}{3} \\
\frac{3}{-}\end{array}$ \\
\hline & Grade 4 & M.D. & $\mathbf{N}$ & 3 & 0 & 4 & 3 & 3 & 0.4 & A & - \\
\hline
\end{tabular}

* Assessment reached after considering clinical and radiological findings.

$\dagger$ Normal first heart sound. Accentuation is graded in four degrees.

The intensity and length of the diastolic murmur did not afford the help that had been expected and a long and loud murmur was frequently heard in patients with severe mitral regurgitation. Nor did the character of the diastolic murmur seem to be distinctive in patients with severe mitral regurgitation and we were seldom able to distinguish in phonocardiograms the different forms of diastolic murmur described by Nixon and Wooler (1960). A third heart sound was rarely heard and seldom recorded in low frequency phonocardiograms. Left ventricular hypertrophy was judged to be present in every patient in whom mitral regurgitation was found but its degree as judged from clinical, radiological, and electrocardiographic findings did not help greatly in assessing the severity of the regurgitation.

The indirect left atrial pressure (wedged pulmonary artery) was increased in every case and the $\mathrm{Ry} / \mathrm{v}$ ratio could be measured in 18 of the 24 patients. Although figures were higher in the patients with more severe regurgitation there was a wide scatter, and a measurement of less than 1.0 found in three patients with grade 3 or 4 mitral regurgitation. 
Comparison of the results with the findings at operation is the most important test of the reliability of such an investigation. Twelve patients have so far undergone mitral valve surgery, 9 with digital exploration and 3 at open cardiotomy. In 9 of the 12 the correlation was exact. In 2 a difference of one grade of regurgitation was found between the two assessments: in one of these grade 3 regurgitation was found when the cardioangiographic assessment had been grade 2, and in the other only grade 1 regurgitation was found when grade 2 had been diagnosed. In a third patient grade 2 mitral regurgitation was found at cardioangiography and this was thought to explain the considerable left ventricular enlargement that was present. She was thought to be suitable for closed valvotomy but at operation there was moderately tight mitral stenosis without detectable regurgitation. This discrepancy remains unexplained.

\section{Complications}

It was found impossible to pass the catheter into the left ventricle in two patients. One of these had aortic regurgitation of moderate degree and the other was thought to have a normal aortic valve. Occasional failure is inevitable but the difficult cases seem impossible to predict. Aortic valve disease, even severe aortic stenosis, does not appear to add greatly to the difficulty of passing the

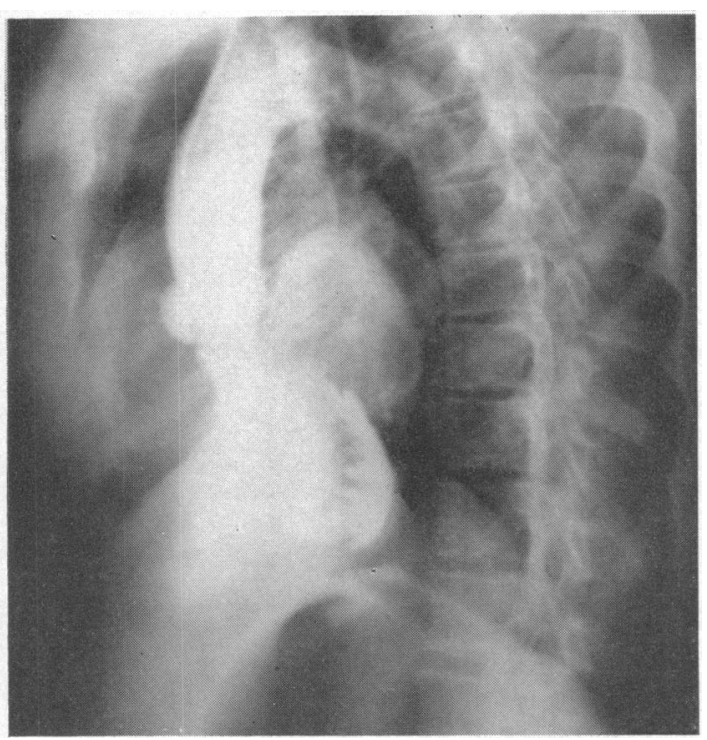

FIG. 7.-Left ventricular cardioangiogram showing contrast medium outlining a portion of the left ventricular wall. catheter into the ventricle. In a third patient in whom difficulty was encountered in passing the aortic valve the catheter tip entered the left coronary artery and caused an immediate ischæmic change in the electrocardiogram. This persisted for several minutes after the catheter was withdrawn and it was thought prudent not to proceed.

Ventricular extrasystoles usually occurred when the catheter entered the left ventricle but ceased after slight adjustment of its position in all but two patients. In these they went on to serious arrhythmias. Ventricular tachycardia developed in one patient and continued for several minutes after the catheter was withdrawn to the aorta. In the second patient ventricular fibrillation occurred: normal rhythm was restored after cardiac massage and defibrillation but irreversible cerebral damage had unfortunately occurred and he died 28 hours later without regaining consciousness. Both these serious ventricular arrhythmias happened when anæsthesia was induced while ventricular ectopic beats were occurring and it seems important that no extra manipulations should be undertaken until the irritation of the ventricle by the catheter is satisfactorily controlled. A similar example of fatal ventricular fibrillation has been described by Amplatz et al. (1961).

A few ventricular ectopic beats commonly occurred as the injection was made. It did not seem to us that these induced fictitious regurgitation as has been suggested (Amplatz et al., 1961). In two patients early in our experience the contrast medium penetrated the myocardium, outlining the pericardium in one instance (Fig. 6) and a portion of the ventricular wall in the other (Fig. 7). In the former no electrocardiographic change occurred but in the latter a very brief ventricular tachycardia was recorded followed by transient bundle-branch block (Fig. 8). Neither patient seemed any the worse for the mishap. Amplatz et al. (1961) describes a fatality in which the catheter tip had entered the left atrium and perforated its wall. Our two cases were probably the result of the catheter tip 


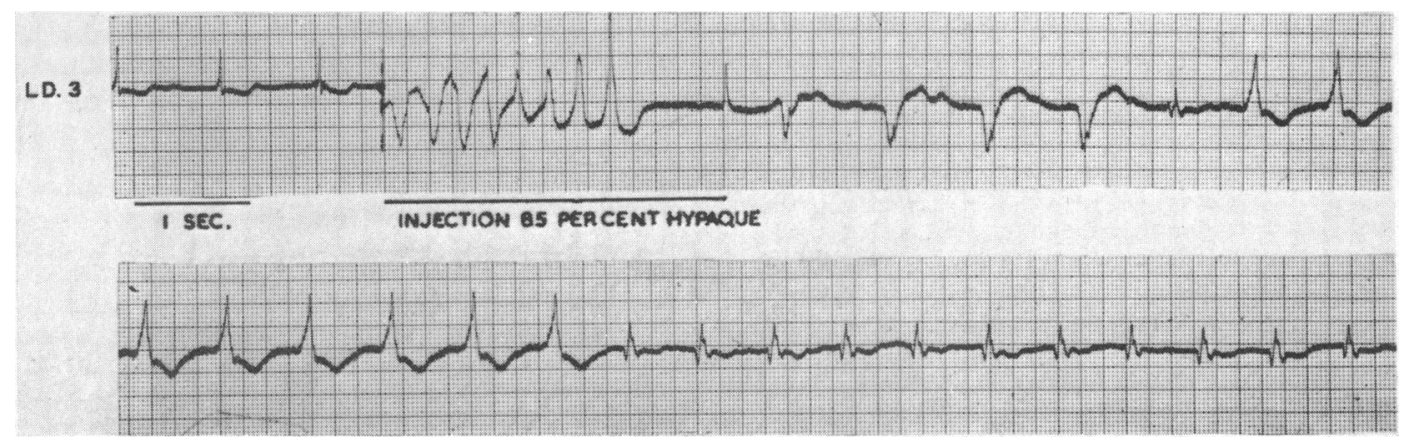

FIG. 8.-Effect on the electrocardiogram of intramural injection of contrast medium (same patient as in Fig. 7). A brief ventricular tachycardia is followed by bundle-branch block of varying form.

lying too far down in the ventricle and touching the endocardium. The straightening of a tortuous catheter as the injection was made may have thrust it harder against the ventricular wall. The opacification of the whole length of the catheter as the preliminary film is taken should lessen the risk of this complication.

\section{DISCUSSION}

The procedure is easily accomplished in most patients even when there is aortic valve disease. Mitral regurgitation is excluded when there is no left atrial opacification after injection of contrast medium into the left ventricle, and when regurgitation is present its degree can be judged with reasonable accuracy, and agrees with the findings at surgical exploration. It has been very helpful in the selection of patients with severe mitral valve disease for surgical treatment. When signs of mitral stenosis and regurgitation coexist closed valvotomy can be recommended if cardioangiography only demonstrates slight regurgitation. If severe regurgitation is shown a closed operation is unlikely to be worth while and open repair of the valve is preferable. Conventional valvotomy has been carried out successfully in all 5 patients operated on who had been shown to have no regurgitation or grade 1 mitral regurgitation. Two patients with grade 3 regurgitation have been operated on by an open procedure and would clearly have been unsuitable for conventional valvotomy. The 5 patients with grade 2 regurgitation who have been operated on present a more difficult problem. In 2 of them conventional valvotomy was successful because the investigation had overestimated the degree of regurgitation. In the other 3 no improvement could be achieved except by an open technique. In our view mitral valvotomy should not be attempted in patients in whom mitral regurgitation of more than grade 1 severity is demonstrated.

The investigation is also helpful in patients with multi-valvular disease when a high indirect left atrial pressure indicates severe mitral valve disease but the physical signs are equivocal. In such patients unsuspected aortic valve disease is sometimes discovered, and mitral regurgitation can be excluded or its severity judged. This is illustrated by the investigation of 2 patients with intractable cardiac failure in whom loud systolic and diastolic murmurs were heard. Tricuspid valve disease was thought to be present, but the systolic murmurs were widely heard and left ventricular enlargement was detected radiologically, so that mitral regurgitation could not be excluded. The Ry/v ratio was $\mathbf{0 . 3 5}$ in one case but the catheter could not be wedged in the pulmonary artery in the other. Left ventricular cardioangiography showed that neither patient had any mitral regurgitation and an unexpected pressure gradient was found at the aortic valve in one patient. Both these patients underwent successful mitral valvotomy.

Comparison of the results of cardioangiography with the clinical findings shows that although severe mitral regurgitation is present where there is a normal or faint first heart sound, a loud mitral 
systolic murmur, and considerable left ventricular hypertrophy, these signs are not always present and in many patients the severity of regurgitation is difficult to judge from clinical examination.

The assessment of mitral regurgitation by comparison of left atrial with aortic opacification as described by Björk et al. (1960) generally agreed with the assessment from left atrial opacification alone. An important discrepancy was, however, observed in two patients (E.T., and I.B., see Table) These patients were judged to have grade 3 mitral regurgitation and yet were in Björk's Group C, that is, the descending aorta was completely filled by the time the left atrium was fully opacified. According to Björk these patients would be suitable for closed valvotomy while in our judgement severe mitral regurgitation is present. Neither patient has yet been operated on so that the discrepancy between the two assessments is unresolved. In our experience Björk's method of assessment is most valuable in the measurement of mitral regurgitation when considered in conjunction with the conventional method: it does not take into account the size of the left atrium or the degree of its opacification and if used alone it could lead to serious misjudgement.

\section{CONCLUSIONS}

A technique of left ventricular cardioangiography by arterial catheterization is described. Thirty patients who were thought to have mitral regurgitation have been investigated by this method, the procedure being accomplished successfully in 24 . The presence of mitral regurgitation could be detected readily and its degree judged from the opacification of the left atrium. The findings on clinical examination and right heart catheterization did not provide an accurate basis for judging the severity of mitral regurgitation. The reliability of the assessment by cardioangiography has been confirmed in 12 patients who have subsequently undergone mitral valve operations.

We are much indebted to Mr. J. L. Collis for his interest in this study and for undertaking the surgical assessment and treatment of these patients. We are also grateful to Dr. J. Wearing and to the staff of the Radiological Department, and to Miss J. Brookes and Mrs. A. Winsor for technical assistance. Miss A. L. Hebden of the Coventry and Warwickshire Hospital kindly prepared the illustrations.

\section{REFERENCES}

Amplatz, K., Lester, R. G., Ernst, R., and Lillehei, C. W. (1961). Radiology, 76, 393.

Arvidsson, H. (1958). Acta radiol. (Stockh.), Suppl. 158.

Björk, V. O., Lodin, H., and Malers, E. (1960). Amer. Heart J., 60, 691.

Brockenbrough, E. C., Braunwald, E., and Ross, J. (1962). Circulation, 25, 15.

Dotter, C. T., and Gensini, G. G. (1960). Radiology, 75, 171.

Lehman, J. S., Musser, B. G., and Lykens, H. D. (1957). Amer. J. Roentgenol., 77, 207.

Nixon, P. G. F., and Wooler, G. H. (1960). Brit. med. J., 2, 1122.

Owen, S. G., and Wood, P. (1955). Brit. Heart J., 17, 41.

Ponsdomenech, E. R., and Beato Núñez, V. (1951). Amer. Heart J., 41, 643.

Prioton, J.-B., Thévenet, A., Pelissier, M., Puech, P., Latour, H., and Pourquier, J. (1957). Presse méd., $65,1948$.

Smith, P. W., Cregg, H. A., and Klassen, K. P. (1956). Circulation, 14, 847. 